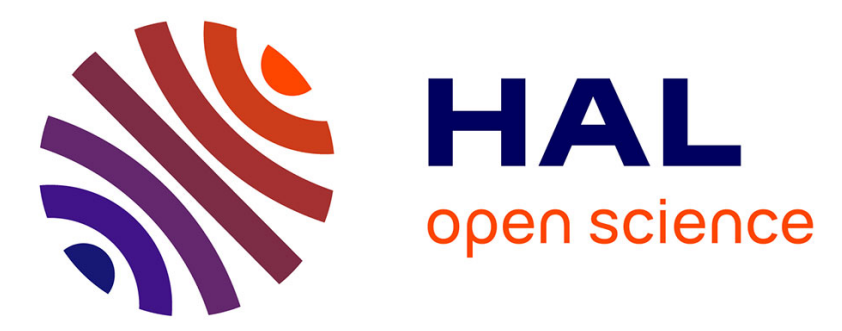

\title{
Physical behavior of electrostrictive polymers. Part 1: Polarization forces
}

\author{
Gildas Diguet, Jean-Yves Cavaille, Gael Sebald, Toshiyuki Takagi, Hiroshi \\ Yabu, Ai Suzuki, Ryuji Miura
}

\section{To cite this version:}

Gildas Diguet, Jean-Yves Cavaille, Gael Sebald, Toshiyuki Takagi, Hiroshi Yabu, et al.. Physical behavior of electrostrictive polymers. Part 1: Polarization forces. Computational Materials Science, 2021, 190, pp.110294. 10.1016/j.commatsci.2021.110294 . hal-03265512

\section{HAL Id: hal-03265512 \\ https://hal.science/hal-03265512}

Submitted on 21 Jun 2021

HAL is a multi-disciplinary open access archive for the deposit and dissemination of scientific research documents, whether they are published or not. The documents may come from teaching and research institutions in France or abroad, or from public or private research centers.
L'archive ouverte pluridisciplinaire HAL, est destinée au dépôt et à la diffusion de documents scientifiques de niveau recherche, publiés ou non, émanant des établissements d'enseignement et de recherche français ou étrangers, des laboratoires publics ou privés. 


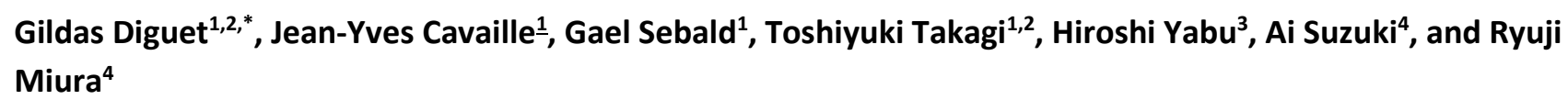

${ }^{1}$ ELyTMaX, CNRS / Université de Lyon / Tohoku University, International Joint Unit UMI 3757, Room 502, 2-1-1 Katahira, Aoba-ku, Sendai 980-8577, Japan

${ }^{2}$ Institute of Fluid Science, Tohoku University, 2-1-1 Katahira, Aoba-ku, Sendai 980-8577, Japan

${ }^{3}$ WPI-Advanced Institute for Materials Research (AIMR), Tohoku University, 2-1-1 Katahira, Aoba-ku, Sendai 980-8577, Japan

${ }^{4}$ New Industry Creation Hatchery Center, Tohoku University, 6-6-10 Aoba, Aramaki, Aoba-ku, Sendai 980-8579, Japan

\section{Abstract}

11 The electrostrictive response of soft elastomers with the same stiffness, strongly depends on their chemical nature 12 and their typically multiphase microstructure. Moreover, some elastomers exhibit a strongly time dependent 13 electrostriction over tens of minutes, and up to now, no theoretical approach has been proposed to analyze 14 experimental data on local parameters like the dielectric constants, conductivities and viscoelastic moduli of these 15 composite-like materials. We consider the phenomenon where the deformation of a polymeric sample between two 16 electrodes is proportional to the square of the applied field, which is known as electrostriction. The electrostatic 17 attraction of charged electrodes is Maxwell electrostriction. In cases, such as block co-polymers with phase 18 separation, the observed electrostriction reaches magnitudes more than 10 times higher than those achieved via the 19 Maxwell process. Phenomenological analyses of experimental data are usually performed but few physical have been 20 proposed to explain the difference. Therefore, we analyze the electric forces inside a composite-like polymer and 21 estimate the corresponding deformation. Using data sets for polyurethane-based materials that exhibit phase 22 separation during their processing, we propose a microstructural model corresponding to a composite where spherical 23 particles randomly fill a matrix. The particles and matrix exhibit different values of physical parameters such as the 24 (i) dielectric constant and electrical conductivity, which determine the local electric field and (ii) viscoelastic 25 modulus, which determine the local stiffness. Because the phases are different, the electric field is not homogenous 26 and the field gradient generates forces around the interfaces. Developing a 2D model, we compare simulation results 27 to experimental literature and other modeling approaches, and discuss them in detail. The polarization forces are 28 found to be responsible for $20 \%$ of the deformation in a material with $35 \%$ inhomogeneity. Though the time constants 29 are consistent with experimental data, their contribution is smaller than the Maxwell contribution, and therefore other 30 mechanisms are involved in the large electromechanical activity of polymers like polyurethanes.

31 *corresponding author: gildas.diguet.d4@tohoku.ac.jp 


\section{Introduction}

When a soft material such as rubber is placed inside an applied electric field $\boldsymbol{E}$ between two electrodes, it also experiences to their electrostatic attraction. This leads in turn to a compressive strain, known as the Maxwell strain, given by [1]

$$
S_{m}=-\left(\frac{\varepsilon}{Y}\right) E^{2}=-M_{m} E^{2}
$$

where $\varepsilon$ and $Y$ are the dielectric constant and Young modulus of the material, and $M_{m}$ the Maxwell electrostrictive coefficient.

However, some electro-active polymers (EAPs) exhibit much higher deformations, $S>>S_{m}$ [2-6]. These high deformations are still quadratic at low applied electric field according to

$$
\mathrm{S}=-\mathrm{M} E^{2}
$$

where $M>M_{m}$ is the electrostriction coefficient. Electrostriction occurs in ionic crystals [7] and is responsible for piezoelectricity in ferroelectrics that have spontaneous polarization [7-9]. Anderson calculated the strain of a homogenous polarizable material in a cubic lattice hence providing a correction to the Maxwell coefficient [10], which Shkel later generalized to the case of amorphous material [11] with corrections based on the dielectric (permittivities), mechanical (Young modulus and Poisson ratio) and structural (lattice) properties.

The electrostrictive response of soft elastomers with the same stiffness strongly depends on their chemical natures, and for multiphase polymers, on their microstructures (such as spherical domains dispersed in continuous matrixes and lamellar materials) [1-6].

Experimental tests of electrostriction can be tricky, and it is difficult to obtain relevant measurements. For example, the constraint induced by the electrodes limits the deformation, as shown by Guyomar et al. [12]. They measured the deformation of $70 \mu \mathrm{m}$ polyurethane (PU) films (i) coated with a $50 \mathrm{~nm}$-thick gold layer and (ii) uncoated. An interferometer was used to measure the electrostrictive coefficients of both coated and uncoated films. Without the gold layer the PU strain went up to $4 \%$, whereas that of the coated film barely reached $1 \%$ for the same applied field of $10 \mathrm{MV} / \mathrm{m}$. The resulting electrostriction coefficients for the coated and the uncoated films were $4.83 \times 10^{-16} \mathrm{~m}^{2} / \mathrm{V}^{2}$ and $1 \times 10^{-16} \mathrm{~m}^{2} / \mathrm{V}^{2}$, respectively. Measurements of the low-field permittivity $\left(\varepsilon=4 \varepsilon_{0}\right)$ and mechanical modulus ( $Y=32 \mathrm{MPa}$ ) lead to $M_{m}=\varepsilon / Y=1.10 \times 10^{-18} \mathrm{~m}^{2} / \mathrm{V}^{2}$. To avoid any measurement artifacts, Zhenyi [13] evaluated the electrostriction coefficient of a PU material using three different experimental apparatuses; the first was an accelerometer, the second an interferometer and the third a capacitance sensor. All measured values were higher than the Maxwell coefficient $\left(1.7 \times 10^{-18} \mathrm{~m}^{2} / \mathrm{V}^{2}\right)$, and the values corrected by removing the Maxwell effect were $M=$ $2.4 \times 10^{-17} \mathrm{~m}^{2} / \mathrm{V}^{2}$ (accelerometer), $3.3 \times 10^{-17} \mathrm{~m}^{2} / \mathrm{V}^{2}$ (interferometer), and $1 \times 63.10^{-17} \mathrm{~m}^{2} / \mathrm{V}^{2}$ (capacitance sensor). Diaconu et al. [6, 14-16] performed several experiments on a PU to study its strong behavior. For example [6], they used a liquid between the metallic electrodes and the polymer film to reduce friction in a Michaelson interferometer when measuring the electrostrictive properties of a $0.05 \mathrm{~mm}$ thick sample with low frequency dielectric constant of $\varepsilon=7.2 \varepsilon_{0}$ and elastic modulus of $Y=13.1 \mathrm{MPa}$. Their measurements yielded $M=7 \times 75.10^{-16} \mathrm{~m}^{2} / \mathrm{V}^{2}$, which is much higher than the simple Maxwell response evaluated at $M_{m}=4.86 \times 10^{-18} \mathrm{~m}^{2} / \mathrm{V}^{2}$. They also obtained similar results, with different film thicknesses [14]. All these data show that additional contribution to electrostriction must be taken into 
account. A phenomenological way to account for this additional contribution is in splitting the deformation coefficient as $M=M_{m}+M_{E}$. If $M_{m}<<M_{E}$, then $M \approx M_{E}$. We hereafter refer to $M_{E}$ as the pure electrostriction coefficient.

This pure electrostriction is an intrinsic function of the material polarization $P$ [17] and is expressed as $S=Q P^{2}$. At low field, $S=Q\left(\varepsilon-\varepsilon_{0}\right)^{2} E^{2}$, yielding $M_{E}=Q\left(\varepsilon^{-} \varepsilon_{0}\right)^{2}$ [17-19]. The quantity $Q$ is proportional to $1 / \varepsilon Y$. Therefore [4, 1721],

$$
M_{E} \approx\left(\varepsilon-\varepsilon_{0}\right) / \varepsilon Y
$$

However, polymers exhibiting $M_{E} \gg M_{m}$ have either been filled with conducting particles or have intrinsic heterogeneities [2, 21, 22-23]. Diblock-copolymers PU, have such intrinsic heterogeneities induced by a phase separation during the sample preparation resulting in a distribution of particles of one phase in a continuous matrix (second phase), for block compositions far from 50\%-50\%. In either case, added particles or a two-phase system, these materials can be viewed as composite materials (particles embedded in a matrix). The microstructural features of diblock-copolymer PUs have been characterized [22].

Some researchers have tried to increase the electrostriction coefficient $M$ by increasing the dielectric constant, such as in the experiments performed by Putson and al. [24] on PU containing polyaniline (PANI). They succeeded in increasing the permittivity $\varepsilon$ with an almost constant modulus as the PANI content increased. Additionally, $M$ for the four tested PU materials was around $10^{-16} \mathrm{~m}^{2} / \mathrm{V}^{2}$ and $M_{m}$ around $10^{-17} \mathrm{~m}^{2} / \mathrm{V}^{2}$.

Although little work has been done at the theoretical level to describe macroscopic behavior using local parameters, it is interesting to note the work of Lefevre and Lopez-Pamies [25], or Stephen et al [26]. They have proposed very rigorous equations and simulations of composites made up of elastomeric matrices incorporating rigid spherical or ellipsoidal particles. In other works, Lefevre and Lopez-Pamies [27-28] have developed a framework devoted to nonlinear elastic behaviour, which must be taken into account for deformations higher than about $1 \%$ for elastomeric polymers: this is the case for the best electroactive materials found in the literature. Such approaches have been developed using sophisticated and elegant homogenization techniques.

The main difference with this work is that they did not take into account electrical conductivity and only considered static conditions.

Diguet et al. [29] examined the idea that these composite-like materials have heterogeneous dielectric properties, which, in turn, locally modify the electric field and create forces. They calculated the force created by a single particle (with dielectric constant $\varepsilon_{2}$ ) embedded in a matrix (with $\varepsilon_{l} \neq \varepsilon_{2}$ ) and estimated the resulting deformation.

Interestingly, these polymers also exhibit a time-dependent electrostriction $S(t)$, as seen in Figure 1. Possible reasons for this behavior are (i) their viscoelasticity and (ii) their dielectric relaxations. Previous works have studied both mechanical [5] and dielectric [30] behaviors for different PU types, and clearly found corresponding relaxation times at room temperature that were lower by more than five orders of magnitude than the typical electrostriction time. This means that, when a force is applied, the polymer deforms in less than a millisecond owing solely to mechanical and dielectric effects. Interestingly, the conductivity relaxation time is on the same order of magnitude observed for $S(t)$. This means that the driving forces are also time-dependent on the order of tens of seconds. In fact, the electric field is governed by two material electrical parameters: permittivity $(\varepsilon)$ and conductivity $(\sigma)$. The EAP polarization 
state depends on the time scale because of charging effects at the electrodes arising from the effective conductivity [5] and dielectric constant. This time-dependent dielectric force or dielectrophoretic (DEP) force used in cellseparator devices or in concentrator devices [31, 32]. This force arises usually from the differences in electric properties $(\varepsilon, \sigma)$ between biological cells and a medium, and depends on the field gradient as well as its frequency. The force experienced by cells is either positive or negative depending on these factors. Although the literature on this topic is abundant $[31,32]$, DEP forces have never been taken into account in elastic soft materials subjected to electric fields.

In this paper, we evaluate the effect of DEP forces as a physical mechanism for pure electrostriction in a compositelike structure. We then calculate the electric field and forces in a polymer with many particles, and obtain the resulting deformation. For diblock-copolymer PU, the phase separation (composition gradient) between domains might not be sharp, so we also evaluated how the profile might influence the macroscopic deformation.

\section{Methods}

\subsection{Materials}

The studied materials were PU materials, provided by Lubrizol Corporation. These PUs are block co-polymers with hard segments (HSs) and soft segments (SSs). HSs are composed of 4.4' methylene bis (phenyl isocyanate) - (MDI) and 1,4-Butanediol (BD), and SSs consist of poly(teramethylene oxide) - (PTMO). Table 1 lists the weight fractions of the three samples studied. The molecular weight of the SSs (PTMO) were $1000 \mathrm{~g} / \mathrm{mol}$ for PU60 and 88, and 2000 $\mathrm{g} / \mathrm{mol}$ for PU75.

Film samples were made via solution casting. The first step was to dissolve each PU in N,N-dimethylformamide (DMF) at $350 \mathrm{~K}$. The solutions were spread onto glass plates using the Doctor Blade applicator (Elcometer®), dried at $340 \mathrm{~K}$ for 1 day and $400 \mathrm{~K}$ for $3 \mathrm{~h}$. The thickness of the dried films was $100 \pm 1 \mu \mathrm{m}$.

Because HSs and SSs are thermodynamically incompatible, a phase separation occurs during their processing, specifically during solvent evaporation. This leads to a rich HS phase or "hard domain" (HD) and a rich SS phase or "soft domain" (SD). If the SS fraction predominates, the material topology is more likely a soft matrix with hard inclusion particles dispersed in it.

Au electrodes were sputtered onto a disk of radius $2 \mathrm{~cm}$ on and below the PU films with a thickness around 10-20 $\mathrm{nm}$. Samples were then placed between the two electrodes and a voltage was applied. The lower electrode was fixed and the top electrode was free to move. The movement of the top electrode was recorded using a double-beam laser based interferometer. Figure 1 shows the strain obtained versus time as a step electric field $E_{0}=2 \mathrm{MVm}^{-1}$ was applied.

These polymers exhibit several relaxations times (Figure 2), which were characterized either with mechanical spectroscopy (using the homemade dynamic mechanical analyzer described in [5]) or through dielectric spectroscopy (Modulab Materials Test System; see [30]). Their relaxation times are compared with the characteristic times associated with electrostriction kinetics. The $\alpha$-relaxation times result from polymer chain movements and impact the mechanical behavior of the PUs as well as their dielectric properties because the chains contain dipoles. The $\alpha$ relaxation times were then determined via dielectric and mechanical measurements [30]; they are at least five orders of magnitude lower than the times associated with electrostriction at room temperature. For reference, we have also 
142 provided the $\beta$ relaxation times in Table 1, although they are slightly weaker than those for $\alpha$. The small PU 143 conductivity, which might originate in ionic diffusion and therefore thermally activated processes, introduces an 144 additional characteristic time on the order of minutes for the three PUs [5]. These two characteristic times, $\alpha$ 145 relaxation (accounting for mechanical and dielectric time effects) and the characteristic time of the conductivity, 146 highlight the importance of conductivity in the time dependence of electrostriction.

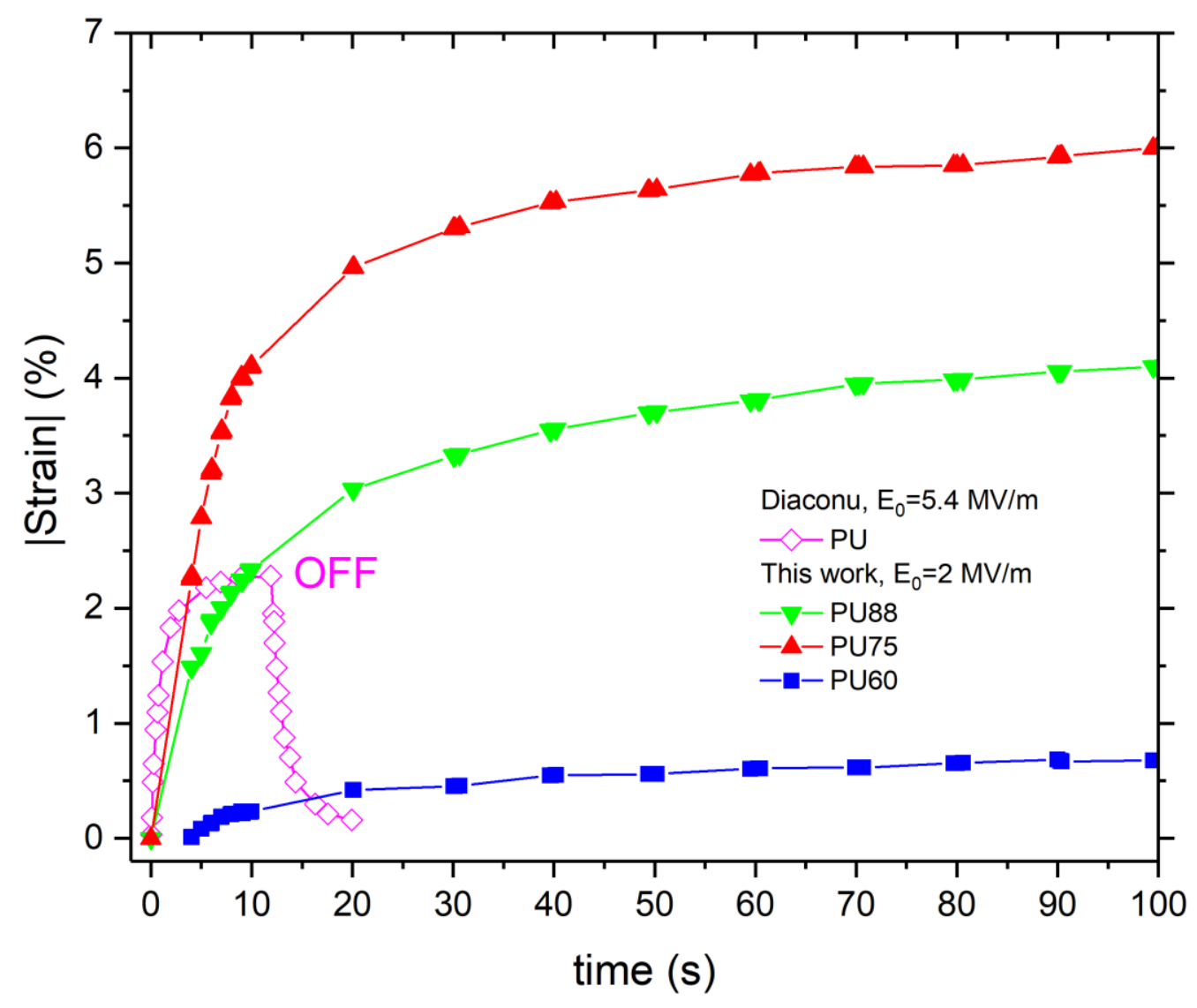

Figure 1. Electro-deformation of polyurethane. The electric field is switched on at time $\mathrm{t}=0$. 
Table 1. PU properties, with hard domain (HD) average diameter $\left\langle\mathrm{D}_{\mathrm{HD}}>\sim 10 \mathrm{~nm}[22]\right.$. Characteristic times $\tau_{e s}, \tau_{\alpha}$ and $\tau_{\beta}$ are for full electrostriction development, and the $\alpha$ - and $\beta$-mechanical relaxations, respectively; $T_{g S D}$ is the glass transition temperature of the soft domains (SDs) of the PU; R.T. refers to room temperature.

\begin{tabular}{|c|c|c|c|c|c|c|c|c|c|c|}
\hline $\mathrm{PU}$ & $\begin{array}{c}\text { Commercial } \\
\text { references }\end{array}$ & $\begin{array}{c}\mathrm{wt} \% \\
\mathrm{HS} \\
{[5]}\end{array}$ & $\begin{array}{c}\text { HD-HD } \\
\text { distance } \\
(\mathrm{nm}) \\
{[18]}\end{array}$ & $\begin{array}{l}T_{g S D} \\
(\mathrm{~K}) \\
{[22]}\end{array}$ & $\begin{array}{c}Y(\mathrm{MPa}) \\
\text { R.T, } \\
0.2 \mathrm{~Hz} \\
{[32]}\end{array}$ & $\begin{array}{c}\varepsilon_{r}(-) \\
\text { R.T, } \\
0.2 \mathrm{~Hz} \\
{[32]}\end{array}$ & $\begin{array}{c}\tau_{e s}(\mathrm{~s}) \\
\text { R.T. } \\
{[5]}\end{array}$ & $\begin{array}{l}\tau_{\alpha}(\mathrm{s}) \\
\text { R.T. } \\
{[30]}\end{array}$ & $\begin{array}{l}\tau_{\beta}(\mathrm{s}) \\
\text { R.T. } \\
{[30]}\end{array}$ & $\begin{array}{c}S / E^{2} \\
\left(10^{-14}\right. \\
\left.\mathrm{m}^{2} / \mathrm{V}^{2}\right) \\
{[5]}\end{array}$ \\
\hline 75 & $\begin{array}{c}\text { Estane X- } \\
4977\end{array}$ & 26 & 17.5 & 205 & 12 & 6.6 & 9 & $10^{-15}$ & $10^{-15}$ & 1.6 \\
\hline 88 & $\begin{array}{c}\text { Estane } \\
58888 \\
\text { NAT021 }\end{array}$ & 45 & 14.3 & 234 & 29 & 7.2 & 15 & $10^{-12}$ & $10^{-18}$ & 1.2 \\
\hline 60 & $\begin{array}{c}\text { Estane } \\
\text { ETE60DT3 } \\
\text { NAT022 }\end{array}$ & 65 & 11.8 & 259 & 201 & 7.0 & 25 & - & $10^{-17}$ & 0.3 \\
\hline
\end{tabular}

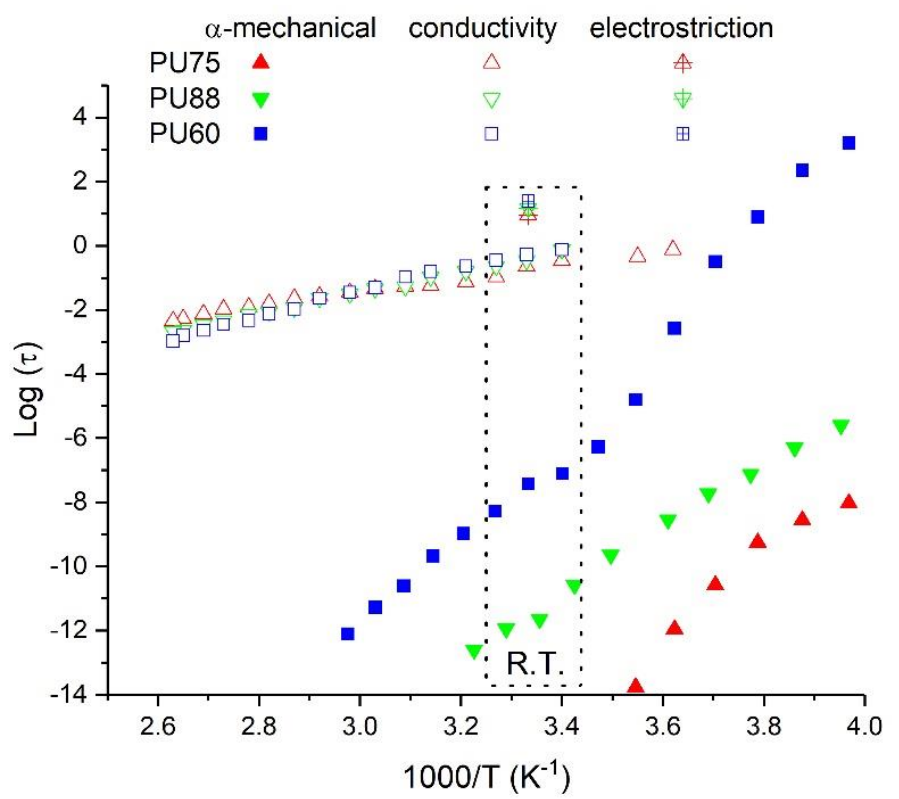

Figure 2. Experimental relaxation times in PUs. Data from $[5,30]$.

\subsection{Simulation}

157 In this paper, the aim is to propose a physical analysis of the mechanisms of mechanical deformation due to the application of electrical forces on the material, and in particular the effect of conductivity (section 3). In this spirit, a material containing a random distribution of identical particles was considered, and the effect of their density and the gradient of their composition at the matrix-particle interface was tested. Several authors have calculated the electrical [34] and mechanical [35] macroscopic properties of similar composites. Such results from a 2D computation are of 
course different from those obtained from a 3D computation, but the trends can be discussed: the influence of the parameters can be compared, and of course, the results are obtained faster than for a 3D computation.

The 2D force calculations were carried out using the "Electric Currents" module of the FEM software COMSOL 5.3. An electric potential was applied to the top electrode, while bottom electrode was grounded electrode, generating an electric field across the materials along the $y$-axis. The local electric field $\vec{E}$, electric polarization field $\vec{P}$, current density $\vec{J}$, and electric displacement field $\vec{D}$, were calculated directly $(\vec{E}=-\vec{\nabla} V, \vec{J}=\sigma \vec{E}, \vec{\nabla} \cdot \vec{J}=0$ and $\vec{D}=\varepsilon \vec{E}=$ $\left.\varepsilon_{0} \vec{E}+\vec{P}\right)$. From these electrical quantities, we derived both the body forces $\overrightarrow{f_{v}}$ in the volume and Maxwell stress $\overrightarrow{f_{s}}$ (see next section), which were then inputted to the "Solid Mechanics" module in COMSOL to obtain the mechanical stress tensor $\overline{\bar{T}}\left(\vec{\nabla} \cdot \overline{\bar{T}}+\overrightarrow{f_{v}}=\overrightarrow{0}\right.$ in the volume and $\overline{\bar{T}} \cdot \vec{n}=\overrightarrow{f_{s}}$ at the electrodes) and the deformation. We also chose the displacement of the top electrode to be along the electric field direction ( $y$-axis), keeping the bottom electrode fixed. The strain was estimated from the displacement of the top electrode. The geometry has been meshed by the COMSOL Solid Mechanics and is composed for a calculation that included 200 particles of 3771110 quadratic triangular elements for a number of degrees of freedom of 22628481.

\section{Electric forces}

\subsection{Maxwell stress}

Maxwell forces cause the attraction of two electrodes with opposite electrical charges. When a dielectric material is placed between the two charged plates, the force experienced depends on the dielectric constant of the medium. The electrode displacement is limited by the countering elastic force of the polymer. The potential $\Delta V$ applied to the electrodes induces a field $E_{0}$ given by $E_{0}=\Delta V / h$ (with $h$ the distance between the electrodes). From theory, the attractive stress acting on each electrode is $f_{s}=\varepsilon E_{0}{ }^{2} / 2$, and the resulting strain is $S_{m}=-(\varepsilon / Y) E_{0}{ }^{2}=-M_{m} E_{0}{ }^{2}$. A typical value of $M_{m}$, for a polymer with $\varepsilon \sim 10 \varepsilon_{0}$ and $Y \sim 1 \mathrm{MPa}$ would be $M_{m} \sim 10^{-17} \mathrm{~m}^{2} / \mathrm{V}^{2}$, which is a thousand times weaker when compared than experimental values (Table 1), $M \sim 10^{-14} \mathrm{~m}^{2} / \mathrm{V}^{2}$.

While the Maxwell effect originates as a stress $f_{s}$ acting on the surface electrodes, we aim to evaluate the contribution of electric body forces (electric forces within the volume).

\subsection{Body forces}

For a polarizable material, the overall force is

$$
\vec{F}=\int_{V} \vec{f}_{v} d V+\oint_{S} \vec{f}_{S} d S
$$

where the electric force per unit volume of a material, with a polarization density $\vec{P}$ can be expressed as

$$
\vec{f}_{v}=(\vec{P} \cdot \vec{\nabla}) \vec{E}
$$

An alternative is to calculate the internal forces in dielectric and magnetic materials. For example, we could use the equivalent charge density to obtain $\vec{f}_{v}=-(\vec{\nabla} \cdot \vec{P}) \vec{E}$. First, the total force $\vec{F}$ is zero if the external field $\vec{E}$ is homogenous. Second, the difference between the two force expressions can be written as a gradient of a scalar 
quantity, which does not change the deformation in an incompressible medium like a polymer well above its glassrubber transition temperature; more details on this topic are in [36-38].

For the PUs studied here, we use the model of a composite material with a host matrix (SD) and filling particles (HD) having different sets of properties $\left(\varepsilon_{1}, \sigma_{1}, Y_{1}\right)$ and $\left(\varepsilon_{2}, \sigma_{2}, Y_{2}\right)$, respectively; we generally take $\varepsilon_{2} \neq \varepsilon_{1}, \sigma_{2} \neq \sigma_{1}$, and $Y_{2} \neq Y_{l}$. Increasing the HS weight percentage slightly increases the SD glass transition temperature $T_{g S D}$ when the phase separation between the SD and HD is not complete, implying that $T_{g S D}$ is much lower than the HD glass transition temperature $T_{g H D}$. The result is that $T_{g S D}<300 \mathrm{~K}<T_{g H D}$, and $Y_{2}>>Y_{l}$ at room temperature. As discussed above, the conductivity of these polymers arises most probably from charge diffusion. This directly implies that, as $Y_{2}>>Y_{1}$, diffusion is much slower in the HD and the conductivities obey $\sigma_{2}<<\sigma_{l}$. Increasing the HS weight percentage decreases the conductivity of the composite, which is consistent with the data in Table 1. Finally, the HD has a higher dielectric constant than the SD because of their chemical structures, and therefore $\varepsilon_{2}>\varepsilon_{1}$.

For the composite, we first calculate the forces exerted on the HD considered as a system of spherical particles, then the forces applied to the SD treated as the host matrix.

3.2.1 Body forces inside the HD. Equation (5) expresses the dielectric force density experienced by a polarized volume in an inhomogeneous electric field. A spherical particle in the HD with given electrical properties $\left(\varepsilon_{2}, \sigma_{2}\right)$ embedded in the SD medium, with electrical properties $\left(\varepsilon_{l}, \sigma_{l}\right)$ is polarized as a function of (i) the applied field $\boldsymbol{E}$, (ii) the particle radius $R$, and (iii) the set of $\varepsilon_{2}, \sigma_{2}, \varepsilon_{l}$, and $\sigma_{1}$. The particle undergoes a DEP force from Eq. (5) as a function of time $[39,40]$ :

$$
F_{D E P}(t)=2 \pi R^{3} \varepsilon_{1}\left[K_{\sigma}\left(1-e^{-t / \tau}\right)+K_{\varepsilon} e^{-t / \tau}\right] \nabla E^{2}
$$

with

$$
K_{\sigma}=\frac{\sigma_{2}-\sigma_{1}}{\sigma_{2}+2 \sigma_{1}}
$$

217 and

$$
K_{\varepsilon}=\frac{\varepsilon_{2}-\varepsilon_{1}}{\varepsilon_{2}+2 \varepsilon_{1}}
$$

$$
\tau=\frac{\varepsilon_{2}+2 \varepsilon_{1}}{\sigma_{2}+2 \sigma_{1}}
$$

The conductivities are contrasted by $K_{\sigma}$ and the permittivities by $K_{\varepsilon}$, and range values from -0.5 (for $\sigma_{1}>>\sigma_{2}$ and $\varepsilon_{1} \gg>\varepsilon_{2}$, respectively) to 1 (for $\sigma_{1}<<\sigma_{2}$ and $\varepsilon_{1}<<\varepsilon_{2}$, respectively). As expected, when $K_{\sigma}$ and $K_{\varepsilon}$ are zero $\left(\sigma_{1}=\sigma_{2}\right.$ and $\varepsilon_{1}=\varepsilon_{2}$, , the polarization force tends towards zero as for homogeneous materials.

Because all materials have finite conductivities, it is important to compare the duration of the experiment with the characteristic time obtained from Eq. (6) [37]. Each of the two exponentially decreasing terms of Eq. (6) is 
dominated by the term with either $K_{\sigma}$ or $K_{\varepsilon}$. Hence, the force is dominated by the $K_{\varepsilon}$ term for times much shorter than $\tau$, and we have

$$
F_{D E P}(t<<\tau)=2 \pi R^{3} \varepsilon_{1} K_{\varepsilon} \nabla E^{2}
$$

In contrast, for times much longer than $\tau$, the force is dominated by the $K_{\sigma}$ term:

$$
F_{D E P}(t>>\tau)=2 \pi R^{3} \varepsilon_{1} K_{\sigma} \nabla E^{2} .
$$

The signs and strengths of the forces change depending on the experimental time, compared with $\tau$. Steady states are dominated by the conductivities, which are limited because $K_{\sigma}$ limits their difference.

3.2.2 Body forces inside soft domains. As pointed out by Diguet et al. [29], a particle with $\varepsilon_{2}>\varepsilon_{1}\left(K_{\varepsilon}>0\right)$ generates a locally inhomogeneous electric field around itself. Similar to Eq. (5), this inhomogeneous electric field induces a body force inside a polarizable medium. The estimate was close to data obtained by extrapolating similar polymer blends, meaning $\varepsilon_{2}>\varepsilon_{1}$. Some elastomeric polymers have relative permittivities of around 10 and are usually considered insulators but, in reality, have low conductivities. Thus, by including the difference in conductivities and permittivities via $K_{\sigma}$ and $K_{\varepsilon}$, we can take time dependence into account for forces such as DEP. For example, following the previous case, where $\varepsilon_{2}>\varepsilon_{1}, \sigma_{2}<\sigma_{1}, K_{\varepsilon}>0$, and $K_{\sigma}<0$, a representative elementary volume of the composite material is a spherical particle as in Fig. 3a, and the matrix experiences a body force depending on its position and time (Fig. $3 b$ and c).

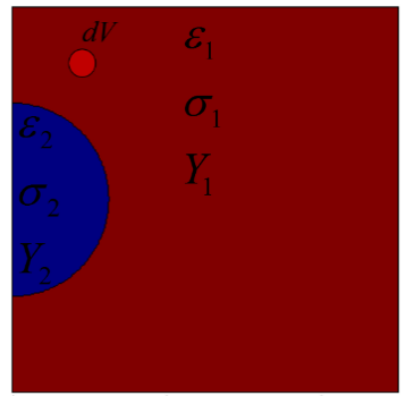

(a)

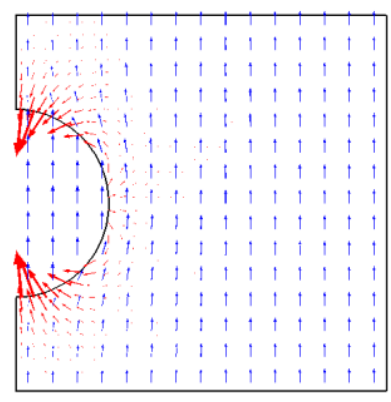

(b)

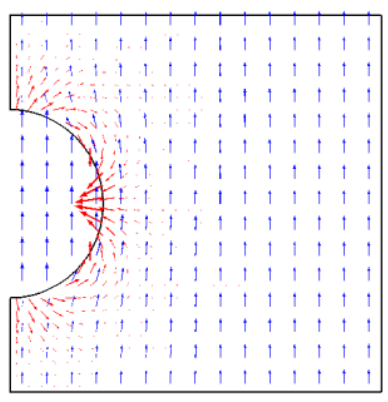

(c)

Figure 3. a) Heterogeneous polymer model. Maps of the body force (red) and electric field (blue) around a particle with properties $\left(\varepsilon_{2}, \sigma_{2}\right)$ in a surrounding medium with properties $\left(\varepsilon_{1}, \sigma_{1}\right)$ with $\varepsilon_{2}>\varepsilon_{1}$ and $\sigma_{2}<\sigma_{1}$ at b) $\mathrm{t}=\tau / 5$ and c) $\mathrm{t}=2 \tau$.

Figure 3 presents map of the electric field and the resulting body force around a spherical particle calculated using Eq. (5) assuming 2D axisymmetry. The forces at a short time, $\tau / 5$ in Fig. 3b, are towards the particle near its top surface and directed outwards at the equator. Moreover, the body forces are stronger near the poles than near the equator. At a longer time, $t=2 \tau$ in fig. $3 \mathrm{c}$, the opposite occurs: the forces are outwards near the top surface of the particle and directed inwards at the equator, and they are stronger near the equator than near the poles. 
In the single-particle picture, the sign of the force experienced by the dipoles in both HD (particle-system) and SD (matrix) depends clearly on the properties of both the HD and SD. The analytical model of a DEP force acting on a sphere helps in understanding forces inside a composite composed of a spherical HD inside an SD: body forces at times $t<\tau$ are dominated by the permittivities, and those at $t>\tau$ are dominated by the conductivities. In the next section, we compute the effects of such time-dependent forces in steady state $(t>>\tau)$ with many particles inside the matrix.

\section{Application to electroactive polymers and discussion}

We next propose a 2D model in which all the above effects are taken into account, namely, (i) the Maxwell forces and (ii) the body forces calculated in the HD and SD. The calculation is performed assuming "linear" materials, meaning that the elastic constants, dielectric constants, and conductivities are independent of the electric field and mechanical strain. Because it is difficult to determine the permittivities of the parent polymers, a preliminary calculation of the electronic distribution of the PU was carried out using tight-binding-based accelerated quantum chemical molecular dynamics [41] at $300 \mathrm{~K}$. This gives average dipole values of 1.18 Debye and 2.40 Debye for PTMO and MDI-BDO, respectively. There is also literature evidence of a larger dipole moment for MDI-BDO than for PTMO [42-44]. These calculations enable the HD and SD permittivities to be evaluated separately. More generally we choose simple parameters to roughly match the effective typical PU values with $\varepsilon=5 \varepsilon_{0}$ to $10 \varepsilon_{0}$ and $Y=1$ $\mathrm{MPa}$ to $10 \mathrm{MPa}$. Parameters for each domain are provided in Table 2. The polymer matrix Poisson ratio is set as 0.49 (very close to typical value of 0.5 ) to avoid volumetric locking during numerical calculation of the mechanical response.

Table 2. Parameter values used for the calculation are typical of the PUs used.

\begin{tabular}{|c|c|c|c|}
\hline & Matrix (1) & Particle (2) & Contrast \\
\hline$\varepsilon\left(\varepsilon_{0} ; \varepsilon_{0}=8.85 \times 10^{-12} \mathrm{~F} / \mathrm{m}\right)$ & 5 & 20 & $K_{\varepsilon}=0.5$ \\
\hline$\sigma(\mathrm{S} / \mathrm{m})$ & $7 \times 10^{-8}$ & $1 \times 10^{-8}$ & $K_{\sigma}=-0.4$ \\
\hline$Y(\mathrm{~Pa})$ & $1 \times 10^{6}$ & $1 \times 10^{9}$ & \\
\hline Poisson ratio $v$ & 0.49 & 0.3 & \\
\hline
\end{tabular}

Lastly, because the contribution of body forces results from electric field gradients, it is important to test the effect of the chemical gradient, near the HD-SD interface. In block copolymers like the PUs used here, the phase separation does not lead to an abrupt change in chemical compositions and therefore of properties, but rather a smooth change in them [45]. This is hence a little different from standard particle-matrix composites and may change the profiles of both the permittivity and conductivity gradients. To account for this peculiar aspect and evaluate its contributions to the body forces, we modeled each particle as a core and boundary with different properties, with intermediate layers (to mimic the interphase) having properties that interpolate smoothly between the core and boundary. The chosen variation function is [46] 


$$
\rho_{i}(\vec{r})=\frac{1}{2}\left[1-\tanh \left(\frac{\left(r-r_{i}\right)-r_{p}}{\alpha}\right)\right]
$$

where $N$ is the number of HD- layers. The properties are plotted in Figure 4

$$
\varepsilon_{\text {composite }}=\varepsilon_{\text {matrix }}+\left(\varepsilon_{\text {particle }}-\varepsilon_{\text {matrix }}\right) \sum_{i=1}^{N} \rho_{i}(\vec{r})
$$
$\varepsilon$ are obtained as spatial functions of the forms

$$
Y_{\text {composite }}=Y_{\text {matrix }}+\left(Y_{\text {particle }}-Y_{\text {matrix }}\right) \sum_{i=1}^{N} \rho_{i}(\vec{r})
$$

$$
\sigma_{\text {composite }}=\sigma_{\text {matrix }}+\left(\sigma_{\text {matrix }}-\sigma_{\text {particle }}\right) \sum_{i=1}^{N} \rho_{i}(\vec{r})
$$

where $\vec{r}$ is the position in the composite (with $r$ the norm of $\vec{r}$ ), $r_{i}$ is the center of the $i$-th HD-layer, $r_{p}=1.5 \mu \mathrm{m}$ is the HD radius, and $\alpha$ is a coefficient setting the particle/matrix interphase size. A small value of $\alpha$ yields a smoother profile, whereas a large value yields a sharper profile. Hence, the Young modulus $Y$, conductivity $\sigma$, and permittivity
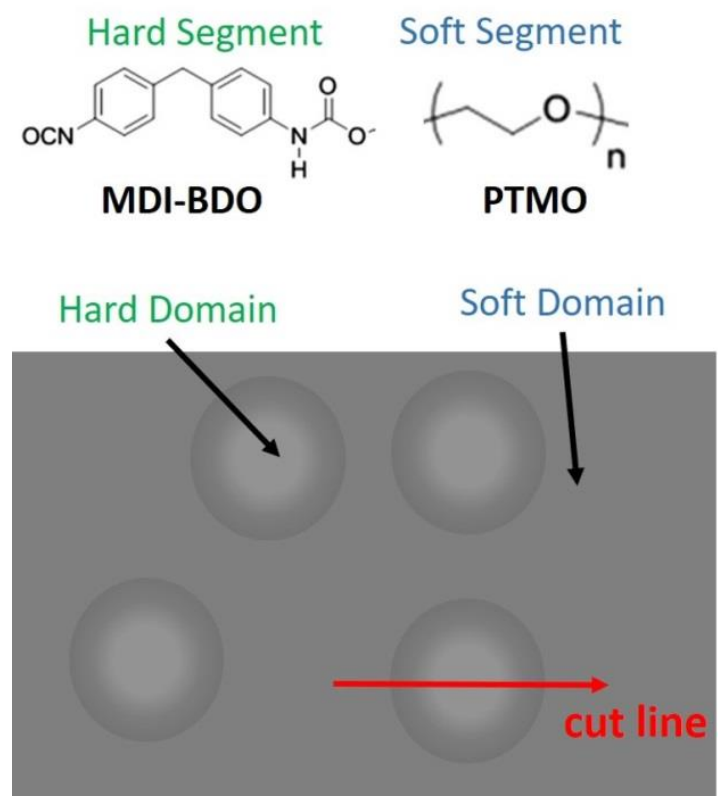

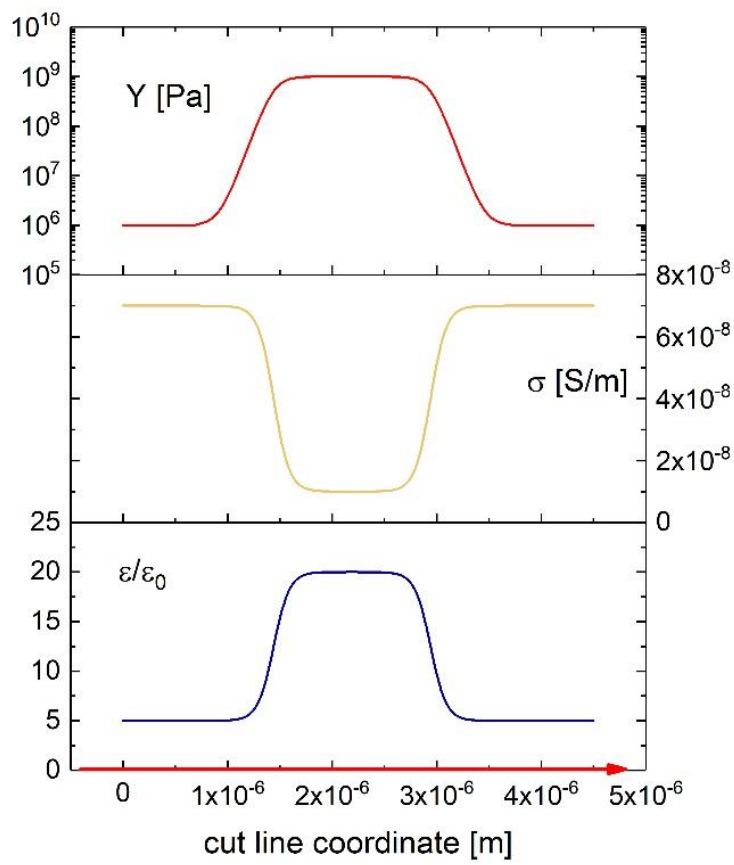

Figure 4. a). PU component and its microstructures. b) Properties along a cut line in the composite material. The two ends of the cut line lie in the matrix, and the line cuts diametrically through the particle. 
A 2D geometry was configured consisting of a rectangle (matrix) with a random distribution of $N$ circular particlelike inclusions, with $N=200$ (Figure 5a). An electric potential was applied so that the field was directed from top to bottom with an average strength $\left|E_{0}\right|=1 \mathrm{MV} / \mathrm{m}$ ( $y$-component) in the steady state (Figure 5a). The electric field $y$ component exhibits some local variation. In particular, the steady state field inside the particles is governed by the conductivity contrast; for example, the field inside a spherical particle is set as $\left|E_{\text {int }}\right|=3\left|E_{0}\right| \sigma_{l} /\left(\sigma_{2}+2 \sigma_{l}\right) \sim 1.5\left|E_{0}\right| \sim$ $1.5 \mathrm{MV} / \mathrm{m}$ if $\sigma_{1}>>\sigma_{2}$. The field is then weaker than $E_{0}$ around the particles The maximum particles fraction of $35 \%$ corresponds to about half of the percolation threshold, which is around $60 \%$ for 2D geometry (compared with $20 \%$ for $3 \mathrm{D}$ ).

Surface: Electric field, y component $(\mathrm{V} / \mathrm{m})$
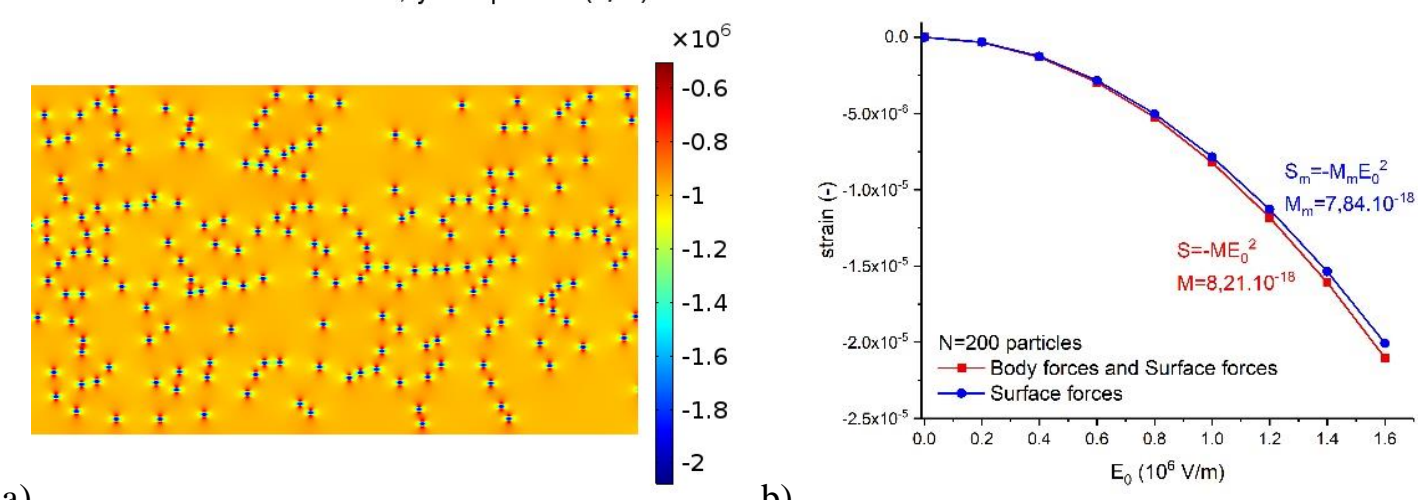

b)

Figure 5. a) Composite-like elastomer: with height $H=10^{-4} \mathrm{~m}(100 \mu \mathrm{m})$ and length $L=2 \times 10^{-4} \mathrm{~m}$ randomly filled with 200 particles $\left(r_{p}=1.5 \mu \mathrm{m}\right)$. The color scale represents the field strength along the direction perpendicular to the electrodes. The applied voltage (top and bottom) was such that the average electric field strength $\left|E_{0}\right|=$ $10^{6} \mathrm{~V} / \mathrm{m}$. b) Effect of the electric field on strain with both body forces and surface forces (red curve) or only surface forces (blue curve).

\subsection{Electrostriction coefficients}

Before analyzing the physical results in more details, it is important to check the influence of the parameters used in this FEM calculation, such as (i) the effect of the random draw of the position of the particles and (ii) their number (at constant volume fraction): in fact, if the results of the calculations depend on the number of particles, we should consider another approach, such as periodic boundary conditions that suppress the edge effect, in order to guarantee reliable results whatever the random draw of the particles dispersed in the matrix [47]. A random dispersion of particles in the matrix leads to many possible distributions which can affect the results, however, for $N=100$ particles with 16 different trials, an average strain $\langle S\rangle=-8.63 \times 10^{-6}$ was obtained with a small a standard deviation of $3 \times 10^{-8}$. A second test was performed with the fraction $\phi=7 \%$ was performed varying the particle number $\mathrm{N}$ (and their radius) from 50 to 450 . Results of this calculation are shown in Figure 6 . Beside, for $\mathrm{N}=75$, the calculation was done 15 times with 15 random distributions of particles. Results seems concentrate in the same range with an average value as $\left(S_{\mathrm{i}}-\langle S(0.07)>) /\left\langle S(0.07)>\sim 5 \times 10^{-3}\right.\right.$, it is then verified that the result of the simulation is mainly 
controlled by the filling fraction and not by the choice of distribution. This shows a consistency of the method to

311 achieve repeatable results.

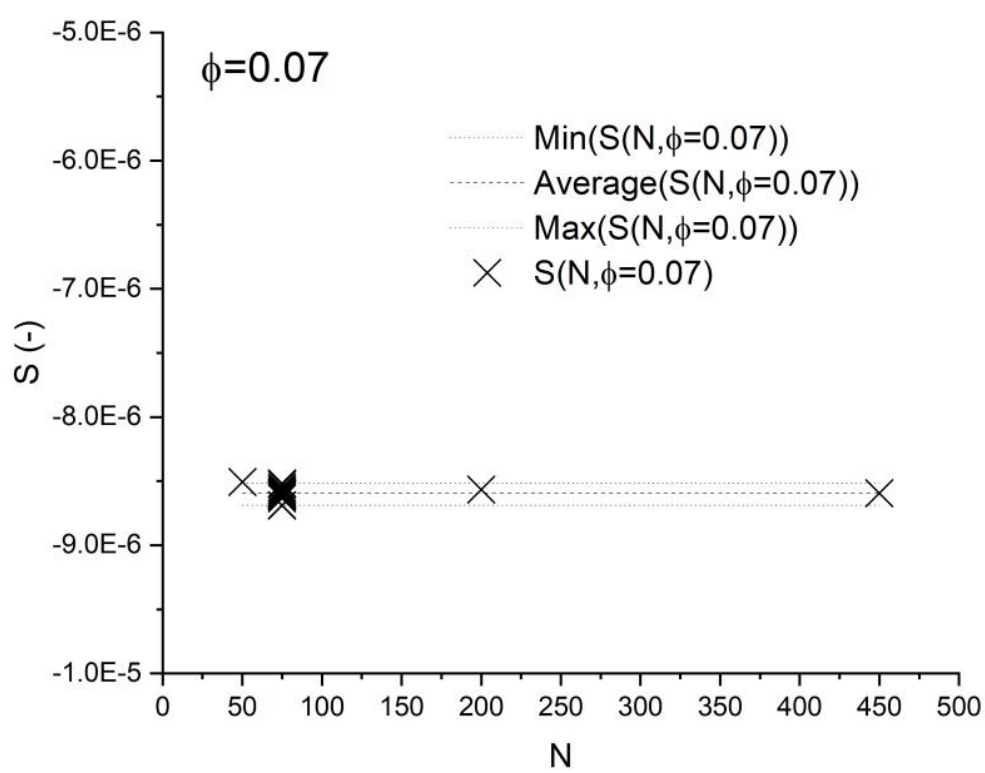

Figure 6. Test of the effect of the number of particles (at constant volume fraction of $\phi=7 \%$ ) on the calculated value of $\mathrm{S}$, and test of different random draw of the position of the particles (for $\mathrm{N}=75$ ).

We calculated the deformation at different applied fields (i) induced by the surface stress only, and (ii) induced by all the forces, as seen in in Figure 5b. For $N=200$ particles; the deformation $S_{m}$ obtained from the Maxwell stress is indicated by the blue dots and curve, while the deformation $S$ obtained from the Maxwell stress and the body force together is indicated by the red dots and curve. As expected, both curves exhibit quadratic behavior with $E_{0}$.

317 Moreover, the deformations are such that $S=-M E_{0}^{2}$ and $S_{m}=-M_{m} E_{0}{ }^{2}$ with $M_{m} \sim 7.84 \times 10^{-18} \mathrm{~m}^{2} / \mathrm{V}^{2}$ and $M$ $318 \sim 8.21 \times 10^{-18} \mathrm{~m}^{2} / \mathrm{V}^{2}$, showing that the body forces slightly reinforce the strain; the composite material is more 319 compressed. This calculation with 200 particles represents a 2D filling factor of nearly 0.07 . In this Figure 7, the effect of particle number, on the pure electrostriction coefficient is presented. At $N=0$, there is only the matrix, and the deformation reaches $S=-M E_{0}{ }^{2}$ with $M=M_{m} \sim 9 \times 10^{-18} \mathrm{~m}^{2} / \mathrm{V}^{2}$ and is less than expected from theory with $\varepsilon / Y$ $=5 \varepsilon_{0} / 10^{6} \sim 45 \times 10^{-18} \mathrm{~m}^{2} / \mathrm{V}^{2}$. This might be an effect of the $2 \mathrm{D}$ geometry and/or of the clamping of the electrodes $[12-13,48]$. The calculated values at $N=0$ are a reference for scaling the effect of particle number. When adding particles, we observe a divergence between $M_{m}$ (Maxwell stress only) and $M$ (Maxwell stress plus body forces), meaning that body forces increase in strength as particle number increases. As discussed above, the electrostriction coefficient $M$ has two contributions: the Maxwell coefficient $M_{m}$ and the pure coefficient $M_{E}$ related to body forces:

$$
S=M E_{0}^{2}=\left(M_{m}+M_{E}\right) E_{0}^{2}
$$




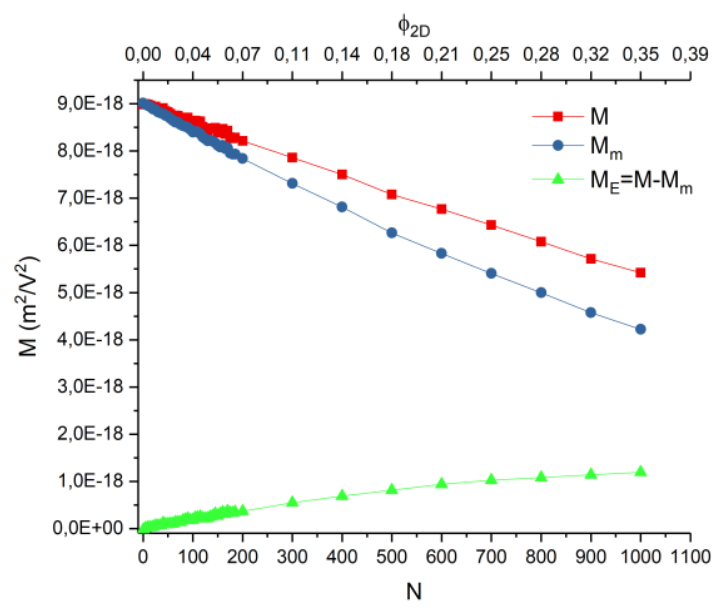

Figure 7. Comparison between the effects of the electrostriction coefficients due to surface and body forces together $(M)$, due to surface force alone $\left(M_{m}\right)$, and due to body force alone $\left(M_{E}\right)$

329 We see in Figure 7 that for $N=1000$ particles, $M \sim 5.4 \times 10^{-18} \mathrm{~m}^{2} / \mathrm{V}^{2}, M_{m} \sim 4.2 \times 10^{-18} \mathrm{~m}^{2} / \mathrm{V}^{2}$, and $M_{E^{\sim}} \sim 1.2 \times 10^{-18} \mathrm{~m}^{2} / \mathrm{V}^{2}$. 330 This means that the body forces are responsible for $22 \%$ of the total calculated deformation. The Maxwell stress is 331 the dominant component. Comparing the value $M \sim 9.0 \times 10^{-18} \mathrm{~m}^{2} / \mathrm{V}^{2}$ obtained with $N=0$ to $M \sim 5.4 \times 10^{-18} \mathrm{~m}^{2} / \mathrm{V}^{2}$ 332 obtained with $N=1000$ particles, the deformation is reduced by $50 \%$ and the percentage of $M_{E}$ in $M$ is increased by $33322 \%$.

3344.2 Effective properties

335 Both coefficients $M_{m}$ and $M$ decrease with increasing particle number, probably because the composite material 336 stiffens as the HD volume fraction increases. Filling the matrix with particles affects the properties of the material 337 such as its dielectric constant, conductivity, and Young modulus. Hence, we extracted these effective values as the 338 particles number increased:

$$
\begin{gathered}
\varepsilon_{e f f}=\langle D\rangle / E_{0} \\
\sigma_{e f f}=\langle J\rangle / E_{0},
\end{gathered}
$$

340 where $\langle q\rangle$ denotes the average of quantity $q$. The effective mechanical modulus of the structure was obtained through 341 numerical experiments by measuring the strain due to a relatively small pressure $\left(Y_{\text {matrix }} / 100\right)$ on the top electrode. 342 Figure 8 shows the effective dielectric constant, conductivity, and Young modulus. 


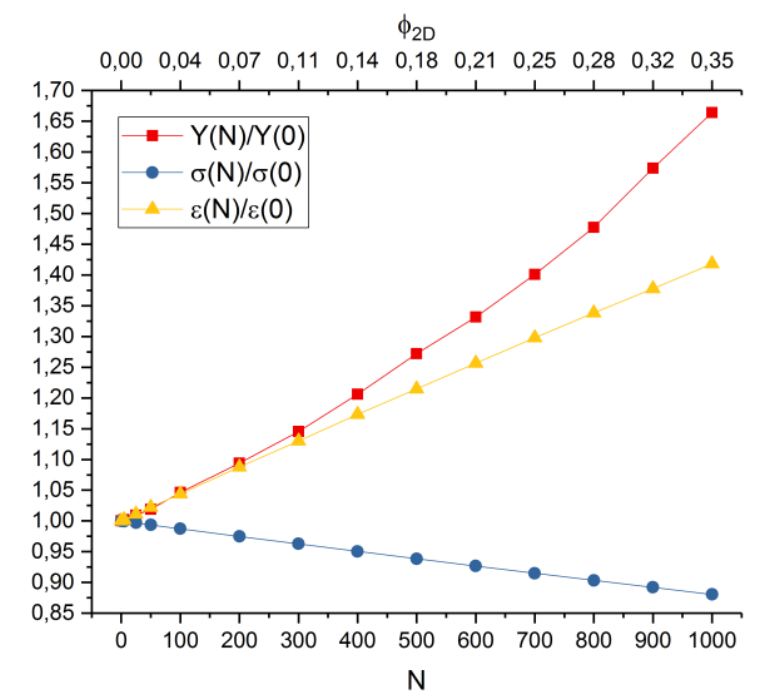

Figure 8. Effective composite properties versus particle number extracted from the $2 \mathrm{D}$ simulation. The values for $N=0$, are those of the matrix (see Table 2).

Clearly, the dielectric constant and Young modulus both increase with increasing $N$ because the particles have higher $\varepsilon$ and $Y$ values than the matrix. In contrast, the effective conductivity decreases because particles have lower conductivities than the matrix. The increase in the Young modulus with particle fraction (HD) is larger than that for the dielectric constant which is experimentally measured in the PU [23]. Moreover, its behavior appears to be nonlinear, whereas the electrical properties look almost linear, at least well below the percolation threshold of the particles.

The spatial distribution of spherical particles in a matrix is known for its electrical properties, which are usually modeled by the Maxwell-Garnett equation [49]:

$$
\frac{\sigma_{e f f}-\sigma_{1}}{\sigma_{e f f}-(d-1) \sigma_{1}}=\varphi \frac{\sigma_{2}-\sigma_{1}}{\sigma_{2}+(d-1) \sigma_{1}}
$$

With $d$ is the dimension of the system, which is the Maxwell-Garnett in 3D $(d=3)$ [50]. The dielectric constant and conductivity follow the Maxwell-Garnett equation. This is usually valid in the dilute regime. A linear behavior of permittivity and conductivity with the volume fraction of particles is then effectively obtained.

The Young modulus, however, follows the non-linear model [51],

$$
\frac{Y_{\text {eff }}}{Y_{\text {matrix }}}=\frac{1}{1-\varphi^{2}}
$$

358 Being a non-linear equation with the filling factor, this explains the larger increase in the Young modulus as the particle number increases comparatively to the electric equations which are linear.

The strains were estimated for the composites. As previously mentioned, $M_{m}=\varepsilon_{\text {eff }} / Y_{\text {eff }}$ decreases as $N$ increases because of the higher increase in the Young modulus with particle fraction than for the dielectric constant. A higher HD content induces a lower strain, which is confirmed by experiment [23]. Moreover, the pure coefficient $M_{E}$ related 
363 to the body forces increases as $N$ increases (Figure 7). To analyze the effect of electrostrictive body forces on the 364 resulting strain, we estimated the equivalent stress by multiplying the strain coefficient $M_{E}$ by the effective Young's 365 modulus and the square of the applied field: $M_{E} Y_{e f f} E_{0}^{2}$. We used the effective Young modulus because we are looking 366 at the displacement of the top surface of the composite material. The result, plotted in Figure 9, shows the equivalent 367 electrostrictive stress exhibiting clearly linear behavior with particle number. Thus, each particle produces an 368 effective stress of $2 \times 10^{-3} \mathrm{~N} / \mathrm{m}^{2}$.

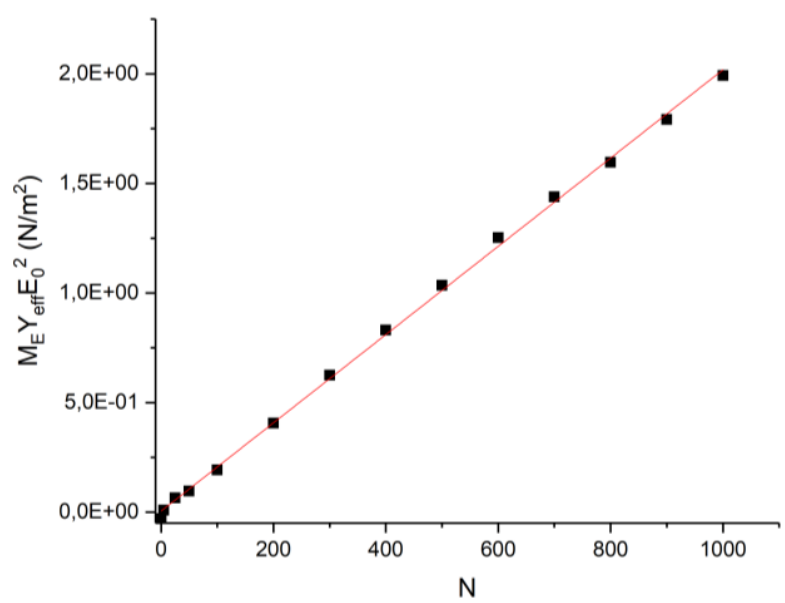

Figure 9. Equivalent electrostrictive stress as a function of the particle number in the matrix.

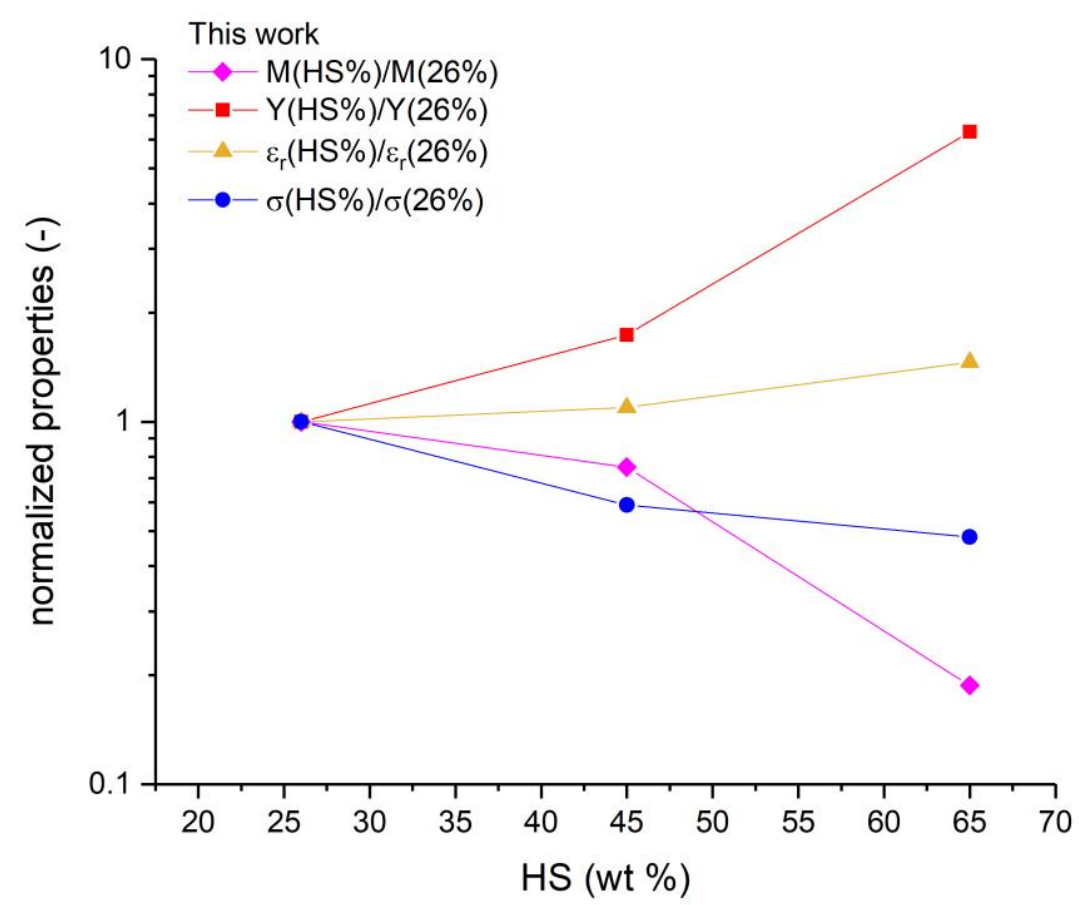

Figure 10. Experimental properties versus HS content of the 3 PUs (see Table 1). 
371 This confirms that increasing the HD fraction reduces $M$ for the reasons presented above. Figure 10 shows the 372 experimental curves of $M, Y, \varepsilon$, and $\sigma$ as functions of HS content, where the HS content (wt $\%$ ) sets the HD fraction 373 inside the material. The experimental properties (Figure 10) behave according to the calculation based on an HD 374 particle in a matrix (Figs. 7-8). In [52-53], two PUs with different HS contents show different experimental 375 deformations for the same applied field of $550 \mathrm{~V} / \mathrm{mm}$ : a measured deformation of $2.11 \times 10^{-3}$ for HS fraction of 42.4 $\mathrm{wt} \%$, and a deformation of $1.39 \times 10^{-3}$ for an HS fraction of $55.2 \%(\mathrm{wt})$. The corresponding mechanical moduli, and conductivities respectively increased and decreased with increasing HS, as in Fig.10. The effect for an SS molecule with different HS contents (wt $\%=20,25,30$, and 35) has been tested previously [23]; the selected SS were: poly(tetramethylene glycol) (PTMG), poly(neopentyl glycol adipate) (PNAD), and poly(dimethyl siloxane) (PDMS). The effect was still as previously found: deformations decreased with increasing HS content independently of the type of SS molecule.

It is interesting to consider other heterogeneous materials, like composites obtained in dispersing conductive particles in a polymeric matrix, and to analyze the role of body forces. For example, a PU filled with particles such as carbon black [54] or carbon nanotube [55] has higher permittivity, conductivity, and mechanical modulus, than a pure PU. As discussed above, the higher permittivity of the particle limits DEP forces. The increase in Young modulus due to the stiff particles causes the deformation first to increase with increasing conductive particle fraction and then decrease when approaching the particle percolation threshold. This is due to the drastic increases in the effective mechanical modulus and electrical conductivity. Thus, the gain expected from the high electrical property values of the fillers disappears.

\subsection{Particle/matrix interface profile effect}

391 Keeping the properties of the matrix and particles constant (Table 2), we changed the value of $\alpha$, which affects the 392 profile of the HD-SD interface. Figure 11 shows various profiles of the relative permittivity gradients, obtained with 393 Eqs. (12-13).

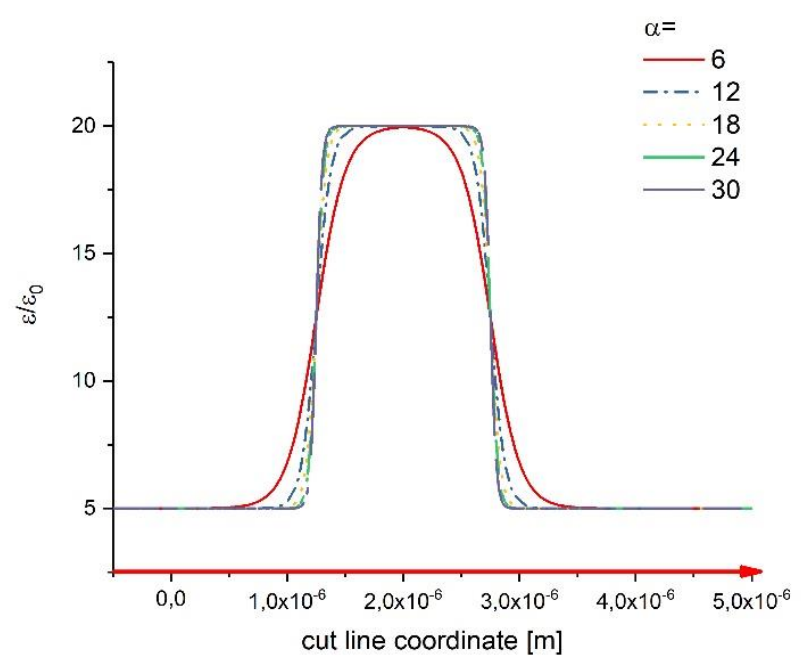

Figure 11. Effect of $\alpha$ on the permittivity along the same cut line as in Fig. 4.

The higher $\alpha$ is, the stronger the dielectric, stiffness, and conductivity gradients are, but in a more confined space. 
mechanical response depends on the effective Young modulus, we calculated the deformation to estimate the resulting electrostriction coefficient for different values of $\alpha$.

Figure 12 shows the effect of the interface thickness on the electrostriction coefficients $M, M_{m}$, and $M_{E}$ along with the effective properties of a composite material with $N=200$ particles.

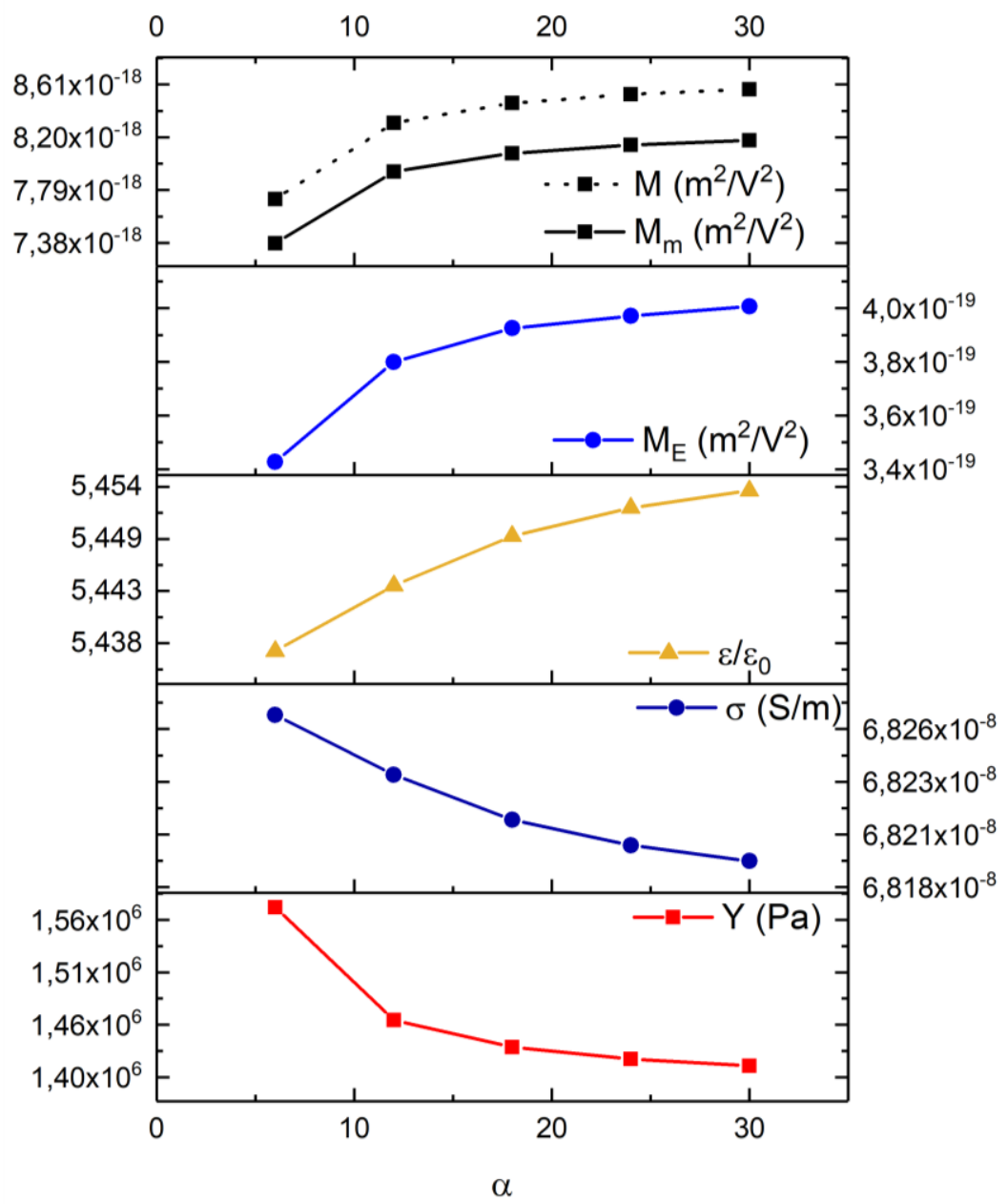

Figure 12. Electrostriction coefficients and effective composite properties as functions of $\alpha$ with $N=200$ particles.

The electrostriction coefficient $M$ increases as $\alpha$ increases; the sharper the interface is, the higher $M$ is. The Maxwell contribution $M_{m}$ increases as $\alpha$ increases. Because $M_{m}$ is proportional to the ratio of the effective permittivity to the effective Young modulus, these two parameters change as $\alpha$ changes. In fact, the effective permittivity increases while the Young modulus decreases, which globally explains the increase in the Maxwell coefficient (see Table 3).

405 Table 3. Effect of $\alpha$ on electrostriction coefficients.

\begin{tabular}{|c|c|c|c|c|c|}
\hline$\alpha$ & $\varepsilon\left(\varepsilon_{0}\right)$ & $Y(\mathrm{MPa})$ & $M_{m}\left(10^{-18} \mathrm{~m}^{2} / \mathrm{V}^{2}\right)$ & $M_{E}\left(10^{-19} \mathrm{~m}^{2} / \mathrm{V}^{2}\right)$ & $M\left(10^{-18} \mathrm{~m}^{2} / \mathrm{V}^{2}\right)$ \\
\hline 6 & 5.43 & 1.57 & 7.37 & 3.44 & 7.72 \\
\hline 30 & 5.45 & 1.41 & 8.17 & 3.96 & 8.57 \\
\hline $\begin{array}{c}\text { Relative } \\
\text { change }\end{array}$ & $0.3 \%$ & $-9.9 \%$ & $10.8 \%$ & $15.1 \%$ & $11.2 \%$ \\
\hline
\end{tabular}


In Table 3, the relative change in $M_{m}$ with $\alpha$ is unsurprisingly about equal to the sum of the relative changes in the dielectrics constant and Young modulus. The relative change in $M_{E}$ with $\alpha$ is the largest, but the values are still lower than those of $M$. For $\alpha=30, M_{E}$ contributes $4.6 \%$ to $M$, while for $\alpha=6$, it is almost the same at $4.5 \%$. The contribution of the electrostriction to the strain does not seem to depend on the property gradients, which is consistent with the fact that increasing $\alpha$ decreases the interphase thickness. The increase in $\varepsilon$ and $\sigma$ gradients is the origin of the body forces.

\section{Conclusion}

The experimental data for some polyurethanes show a typical electrostriction coefficient on the order of $10^{-14} \mathrm{~m}^{2} / \mathrm{V}^{2}$ and Maxwell strain on the order of $10^{-17} \mathrm{~m}^{2} / \mathrm{V}^{2}$. In this study, we calculated PU deformation with a polarization contribution from heterogeneities in the PU after a partial phase separation. The DEP forces, which are timedependent body forces, contribute additionally to the deformation; the Maxwell contribution is a surface force acting on the electrodes. The deformation is complete a few tens of seconds after the voltage is applied because the electrical conductivity agrees with experimental data. The pure electrostriction coefficient of these body forces is responsible for up to $22 \%$ of the total deformation in the range of our calculation (up to a maximal 2D filling of $35 \%$ ). Increasing the particle volume fraction above $35 \%$ (2D) is ineffective because a strong stiffening occurs in the composite material. The profile modifications of the electrical permittivity, electrical conductivity, and mechanical stiffness between stiff particles (HD) and the matrix (SD) also induce a change in the electrostriction coefficient, but this change remains on the same order of magnitude. Furthermore, the DEP magnitude is limited at short times by the permittivity contrast factor $K_{\varepsilon}$ and at long times by the conductivity contrast factor $K_{\sigma}$. It is not possible to enhance the electrostriction coefficient by a factor higher than a few units just by changing the HD permittivity and/or conductivity in two-phase polymers such as a PU or by adding high permittivity/conductivity particles to that polymer.

Our numerical simulations were first performed in as simple a framework as possible, i.e. with a limited number of particles, no periodic boundary conditions, and considering only linear constitutive behaviors, and we obtained results much smaller than the experimental data, at equivalent particle volume fraction, but with a large variation in the number of particles. Consequently, we can assume that the contribution of the polarization body force is too low to explain the discrepancy between the deformation measurements and the Maxwell strain among the possible scenarios. More investigations are needed to understand the large electrostriction observed in various copolymer-like PUs. It is noteworthy that recent works are focused on time dependent phenomena at the origin of polymer electroactivity [56-57]. A very interesting approach by Gosh et al., consists in showing that the dielectric constant drastically increases at decreasing frequency, i.e. increases as a function of time when an electric field step is applied to the material [56]. On the other hand, the electrical conductivity observed in our materials indicates that electrical charges are free to diffuse inside elastomers, and therefore the space charges are to be taken into account as possible contributions to the deformation as shown by some recent works [58]. Following such consideration, we are currently investigating the electrostatic interactions of more or less mobile electrical charges responsible for the above conductivity, and will present it in Part 2 of this work. 


\section{Acknowledgments}

This work was partly supported by the Japan Society for the Promotion of Science (JSPS) Core-to-Core Program, A. Advanced Research Networks, "International research core on smart layered materials and structures for energy saving”. We thank Mark Kurban, from Edanz Group (www.edanzediting.com/ac) for editing a draft of this manuscript.

\section{References}

[1] R.E. Pelrine, R.D. Kornbluh, Electrostriction of polymer dielectrics with compliant electrodes as a means of actuation, Sensors and Actuators A: Physical 64 (1998) 177-85.

[2] D. Jaaoh, C. Putson, N. Muensit, Contribution of Electrostriction in polyurethane/polyaniline blends, Advanced Materials Research,1025-1026 (2014) 697-702.

[3] J.Su, Q.M. Zhang, C.H. Kim, R. Capps, Effects of transitional phenomena on the electric field induced strainelectrostrictive response of a segmented polyurethane elastomer, J. Appl. Polym. Sci. 65 (1997) 1363-1370.

[4] F.M. Guillot, E. Balizer, Electrostrictive effect in polyurethanes, Applied Polymer 89 (2003) 399-404.

[5] M.H. Jomaa, L. Seveyrat, V. Perrin, L. Lebrun, K. Masenelli-Varlot, G. Diguet, J.Y. Cavaille, Difference between electrostriction kinetics, and mechanical response of segmented polyurethane-based EAP, Smart Mater. Struct. 26 (2017) 035049.

[6] I. Diaconu, D.O. Dorohoi, F. Topoliceanu, Electrostriction of a polyurethane elastomer-based polyester, IEEE Sensors Journal 6 (2006) 876-880.

[7] F. Li, L. Jin, S. Zhang, Electrostrictive effect in ferroelectrics: An alternative approach to improve piezoelectricity. Appl. Phys. Rev. 1 (2014) 011103.

[8] I. Katsouras, K. Asadi, M. Li, T.B. van Driel, K.S. Kjær, D. Zhao, T. Lenz, Y. Gu, P.W.M. Blom, D. Damjanovic, M.M. Nielsen, D.M. de Leeuw, The negative piezoelectric effect of the ferroelectric polymer poly(vinylidene fluoride), Nature Materials, 15 (2016) 78-84.

[9] T. Fukukawa, N. Seo, Electrostriction as the origin of piezoelectricity in ferroelectric polymers, Jpn. J. Appl. Phys. 29 (1990) 675.

[10] R. A. Anderson, Mechanical stress in a dielectric solid from a uniform electric field, Phys. Rev. B 33 (1986) 1302.

[11] Y.M. Shkel, D.J. Klingenberg, Electrostriction of polarizable materials: Comparison of models with experimental data, Journal of Applied Physics 80 (1996) 4566.

[12] D. Guyomar, K. Yuse, P.J. Cottinet, M. Kanda, L. Lebrun, Focus on the electrical field-induced strain of electroactive polymers and the observed saturation, J. of Applied Physics 108 (2010) 114910.

[13] M. Zhenyi, J.I. Scheinbeim, J.W. Lee, B.A. Newman, High field electrostrictive response of polymers, J. Polymer Science: Part B: Polymer Physics 32 (1994) 2721-2731.

[14] I. Diaconu, D.O. Dorohoi, C. Ciobanu, Electromechanical response of polyurethane films with different thickness, Rom. Journ. Phys. 53 (2008) 91-97. 
[15] I. Diaconu, D.O. Dorohoi, Properties of polyurethane thin films, J. of Optoelectronics and Advanced Materials 7 (2005) $921-924$.

[16] I. Diaconu, D.O. Dorohoi, An experimental investigation of electroactive polyurethane, Journal of Optoelectronics and Advanced Materials 7 (2005) 2797 - 2801.

[17] B. Guiffard, L. Seveyrat, G. Sebald, D. Guyomar, Enhanced electric field-induced strain in non-percolative carbon nanopowder/polyurethane composites, J. Applied Physics D: Applied Physics 39 (2006) 3053-3057.

[18] K. Wongtimnoi, J.Y. Cavaille, J.M. Chenal, B. Guiffard, A. Bogner, L. Seveyrat, Thickness-dependent microstructural and electromechanical properties in polyurethane films obtained by polymer solution casting, $\mathrm{J}$. of Applied Polymer Science 135 (2018) 46981-46989.

[19] D. Guyomar, P.J. Cottinet, L. Lebrun, C. Putson, K. Yuse, M. Kanda, Y. Nishi, The compressive electrical field electrostrictive coefficient M33 of electroactive polymer composites and its saturation versus electrical field, polymer thickness, frequency, and fillers, Polym. Adv. Tech. 23 (2012) 946-950.

[20] L. Lebrun, D. Guyomar, B. Guiffard, P.J. Cottinet, C. Putson, The characterization of the harvesting capabilities of an electrostrictive polymer composite, Sensors and Actuators A 153 (2009) 251-257.

[21] M. Roussel, C. Malhaire, A.L. Deman, J.F. Chateaux, L. Petit, L. Seveyrat, J. Galineau, B. Guiffard, C. Seguineau, J.M. Desmarres, J. Martegoutte, Electromechanical study of polyurethane films with carbon black nanoparticles for MEMS actuators, J. Micromech. Microeng. 24 (2014) 055011.

[22] M.H. Jomaa, K. Masenelli-Varlot, G. Diguet, L. Seveyrat, L. Lebrun, K. Wongtimnoi, C. Vechambre, J.M. Chenal, J.Y. Cavaille, Modeling of segmented pure polyurethane electrostriction behaviors based on their nanostructural properties Polymer 62 (2015) 139-147.

[23] N.J. Jo, D.H. Lim, G.M. Bark, H.H. Chun, I.W. Lee, H. Park, Polyurethane-based actuators with various polyols, J. Mater. Sci. Technol., 26 (2010) 763-768.

[24] C. Putson, D. Jaaoh, N. Muensit, Large electromechanical strain at low electric field of modified polyurethane composites for flexible actuators, Materials Letters 172 (2016) 27-31.

[25] V. Lefèvre, O. Lopez-Pamies, The overall elastic dielectric properties of a suspension of spherical particles in rubber: An exact explicit solution in the small-deformation limit, Journal of Applied Physics 116, 134106 (2014).

[26] S. A. Spinelli, V. Lefèvre, O. Lopez-Pamies, Dielectric elastomer composites: A general closed-form solution in the small-deformation limit, Journal of the Mechanics and Physics of Solids 83 (2015) 263-284.

[27] V. Lefèvre, O. Lopez-Pamies, Nonlinear electro-elastic deformations of dielectric elastomer composites: I Ideal elastic dielectrics, Journal of the Mechanics and Physics of Solids 99 (2017) 409-437.

[28] V. Lefèvre, O. Lopez-Pamies, Nonlinear electro-elastic deformations of dielectric elastomer composites: II Non-Gaussian elastic dielectrics. Journal of the Mechanics and Physics of Solids 99 (2017) 438-470.

[29] G. Diguet, A. Bogner, J.M. Chenal, J.Y. Cavaille, Physical modeling of the electromechanical behavior of polar heterogenous polymers, J. Applied Physics 112 (2012) 114905.

[30] M.H. Jomaa, L. Seveyrat, L. Lebrun, K. Masenelli-Varlot, J.Y. Cavaille, Dielectric properties of segmented polyurethanes for electromechanical applications Polymer 63 (2015) 214-221. 
[31] C. Zhang, K. Khoshmanesh, A. Mitchell, K. Kalantar-zadeh, Dielectrophoresis for manipulation of micro/nano particles in microfluidic systems, Anal. Bioanal. Chem. 396 (2010) 401-420.

[32] B. Simmons, G. McGraw, R. Davalos, G. Fiechtner, Y. Fintschenko, E. Cummings, The Development of Polymeric Devices as Dielectrophoretic Separators and Concentrators, MRS Bulletin 31 (2006) 120-124.

[33] K. Wongtimnoi, Polyurethane electrostrictifs et nanocompoites : caracterisation et analyse des mecasnismes de couplages electromecaniques, (2011) Thesis.

[34] K.Y. Huang, L.S. Schadler, Understanding the strain dependent dielectric behavior of carbon black reinforced natural rubber- An interfacial or bulk phenomenon. Composites Science and Technology 142 (2017) 91-97.

[35] Z. Wang, D.R. Schmitt, R. Wang, Modeling of viscoelastic properties of nonpermeable porous rocks saturated with high viscous fluid at seismic frequencies at the core scale. Journal of Geophysical Research : Solid Earth 122 (2017) 6067-6086.

[36] H.S. Choi, S.H. Lee, I.H. Park, General formulation of equivalent magnetic charge method for force density distribution on interface of different materials, IEEE: Transactions on Magnetics 41 (2005) 1420-1423.

[37] M. Zahn, Derivation of the Korteweg-Helmholtz electric and magnetic force densities including electrostriction and magnetostriction from the quasistatic Poynting's theorem, 2006 IEEE Conference on Electrical Insulation and Dielectric Phenomena, (2006) 186-189.

[38] M. Petit, A. Kedous-Lebouc, Y. Avenas, M. Tawk, E. Artega, Calculation and analysis of local magnetic forces in ferrofluids, Przeglad Elektrotechniczny (Electrical Review), 87 (2011) 115-119.

[39] L. Benguigui, I.J. Li, More about the dielectrophoric force, J. Appl. Phys. 53 (1982) 1141-1143.

[40] T.B. Jones, Dielectrophoretic force calculation, J. Electrostatics, 6 (1979) 69-82.

[41] F. Ahmed, R. Nagumo, R. Miura, A. Suzuki, H. Tsuboi, N. Hatakeyama, H. Takaba, A. Miyamoto, CO Oxidation and NO Reduction on a $\mathrm{MgO}(100)$ Supported Pd Cluster: A Quantum Chemical Molecular Dynamics Study, J. Phys. Chem. C 115 (2011) 24123-24132.

[42] R.V. Law, Y. Sasanuma, Conformational Characteristics of Poly (tetramethylene oxide), Macromolecules 31 (1998) 2335-2342.

[43] C. Zhang, J. Hu, S. Chen, F. Ji, Theoretical study of hydrogen bonding interactions on MDI-based polyurethane, J. Molecular Modeling 16 (2010) 1391-1399.

[44] M. Ramirez, J. Vargas, M Springborg, Energetic, Structural, and Vibrational Properties of 4,4'Methylenediphenyl Diisocyanate with Relevance for Adhesion, J. Phys. Chem. A 120 (2016) 4256-4266.

[45] C.G. Seefried, J.V. Koleske, F.E. Critchfield, Thermoplastic urethane elastomers. II. Effects of variations in hard-segment concentration, J. Applied Polymer Science 19 (1975) 2503-2513.

[46] J. Merlin, J.F.L. Duval, Electrodynamics of soft multilayered particles dispersions: dielectric permittivity and dynamic mobility, Phys. Chem. Chem. Phys. 16 (2014) 15173-15188.

[47] A.A. Gusev, Representative volume element size for elastic composites: a numerical study, J. of the Mech. and Phys. of Solids 45 (1997) 1449-1459.

[48] H. Wang, Q.M. Zhang, L.E. Cross, A.O. Sykes, Clamping effect on the piezoelectric properties of poly(vinylidene fluoride-trifluoroethylene) copolymer, Ferroelectrics 150 (1993) 255-266. 
554 [49] S. Torquato, Random Heterogeneous Materials: Microstructure and Macroscopic Properties Appl. Mech. Rev. 55 (2002), B62-B63.

556 [50] R. Ruppin, Evaluation of extended Maxwell-Garnett theories, Optics Communications 182 (2000) 273-279.

557 [51] O. Lopez-Pamies, An exact result for the macroscopic response of particle-reinforced Neo-Hookean solids. Journal of Applied Mechanics 77 (2010) 021016.

559 [52] K. Petcharoen, A. Sirivat, Electrostrictive properties of thermoplastic polyurethane elastomer: Effects of urethane type and soft-hard segment composition, Current Applied Physics 13 (2013) 1119-1127.

[53] H.O. Lim, G.M. Bark, H. Park, H.H. Chun, N.J. Jo, High-Field Maxwell Stress Effect of Dielectric Actuator Based on Segmented Polyurethane, Adv. Mat. Research 26 (2007) 753-756.

[54] K. Wongtimnoi, B. Guiffard, A. Bogner-Van de Moortèle, L. Seveyrat, C. Gauthier, J.Y. Cavaillé, Improvement of electrostrictive properties of a polyether-based polyurethane elastomer filled with conductive carbon black, Composites Science and Technology 71 (2011) 885-892.

[55] K. Wongtimnoi, B. Guiffard, A. Bogner-Van de Moortèle, L. Seveyrat, J.Y. Cavaillé, Electrostrictive thermoplastic polyurethane-based nanocomposites filled with carboxyl-functionalized multi-walled carbon nanotubes (MWCNT-COOH): Properties and improvement of electromechanical activity, Composites Science and Technology 85 (2013) 23-28.

[56] Chatchai Putson, Darika Jaaoh, and Nantakan Muensit, Interface Polarization Effect on Dielectric and Electrical properties of Polyurethane (PU)/Polyaniline (PANI) Polymer Composites, Advanced Materials Research Online: ISSN: 1662-8985, Vol. 770, pp 275-278, doi:10.4028/www.scientific.net/AMR.770.275

[57] Kamalendu Ghosh, Jinlong Guo, Oscar Lopez-Pamies, Homogenization of time-dependent dielectric composites containing space charges, with applications to polymer nanoparticulate composites, International Journal of Non-Linear Mechanics 116 (2019) 155-166

[58] V. Lefèvre, O. Lopez-Pamies, Homogenization of elastic dielectric composites with rapidly oscillating passive and active source terms. SIAM Journal on Applied Mathematics 77 (2017) 1962-1988. 